\title{
Eine Bemerkung über die Berechnung der Refraction.
}

Die meisten Theorien der Refraction begnügen sich mit der Integration des Ausdruckes

$$
\frac{a}{1-\alpha} \frac{\sin z d w}{\left\{\cos z^{2}-2 a w+2 s \sin z^{2}\right\}^{\frac{1}{2}}}
$$

indem die Bemerkung gemacht wird, dass das folgende Glied

$$
-\frac{a}{1-a} \frac{s \sin z\left\{\cos z^{2}-2 a w\right\}+\frac{3}{2} s^{2} \sin z^{3}}{\left\{\cos z^{2}-2 a w+2 s \sin z^{2}\right\}}
$$

nichts Merkbares hinzufügt; dies ist aber in Rücksicht auf die Genauigkeit der gegenwartigen Beobachtungen nicht völlig zutreffend; man hat sich wohl zu diesem Feblschlusse dadurch rerleiten lassen, dass dieses Glied selbst in der Horizontalrefraction nur $-\mathrm{l}^{\prime \prime} 4$ beträgt und gemeint, dass dasselbe Glied ebenso rasch versclwindet mit der Zunahme der $\mathrm{Höhe}$ wie die übrigen Korrectionsglieder; das ist aber nicht richtig. Für deu mittleren Zustand der Atmosphare wird das erste Glied der Entwickelung (ausreichend bis $80^{\circ}$ Zenithdistanz) ergeben:

$$
\text { - 0"073 tg. " , }
$$

tritt also in sehr genauen Beobachtungsreihen, wie \%. B. bei den Peters'schen Beobachtungen am Pulkowaer Vertikalkreise sehr merkbar hervor. Es ist übrigens klar, dass sich dieses Glied bei der Bestimmung der Konstante der Refraction aus den Beobachtungen, wenn man nicht allzu grosse Zenithdistanzen heranzieht, mit derselben vereinigt, also bei der Darstellung der Beobachtungen keinen wesentlichen Felıler aus dieser Uebergehung entstehen; es erscheint nur die Konstante um Etwas felılerhaft bestimmt.

Wien 5. Febr. 1878.

\section{Anmerkung über den Doppelstern 2 547, von Prof. Winmecke.}

In Nr. 2176 dieser Zeitschrift lenkt Mr. Burnham die Aufmerksamkeit der Astronomen auf den Doppelstern $\Sigma$ 547. Da derselbe sich nicht in den „Positiones mediae" findet, so gehïrt er $\% \mathbf{u}$ denjenigen Struve'schen Doppelsternen, deren Beobachtung, zur Ausfüllung der allmälig entstchenden Lücken bei der Wiederbeobachtung der Bradley'schen Sterne mittelst des Repsold'schen Meridiankreises der Pulkowacr Sternwarte, ich mir im Jahre 1862 vorgesetat hatte. Ich hatte daker in dem vor kurzem erschienenen siebenten Bande der "Observations de Poulkova", welcher die ron mir in den Jahren 1858-1864 angestellten Meridiankreisbeobachitungen enthält, dieseu Stern aufgesucht und gefunden, dass derselbe 1862 Nor. 19 und 1863 Oct. 6 beobachtet ist. Am ersten Tage fehlt die Rectascension ; eine Aumerkung lautet: "le satellite est soupçonnée dans la direction $P=0^{0.6}$; diese Schätzung kommt recht gut mit den von Herrn Burnham mitgetheilten Messungen uberein. Durch die auf das Erkennen des schwierig wahrnelımbaren Begleiters verwandte Aufmerksamkeit erklärt sich auch, weshalb die Rectascension an diesem Tage fehlt. Die beiden Beobachtungen geben:

$$
\text { Rectasc. 1863.0 Decl. } 1863.0
$$

1862 Nor. $19 \quad-\quad-1^{\circ} 40^{\prime} 32^{\prime \prime 2}$

1863 Oct. $64^{\mathrm{h}} 18^{\mathrm{m}} \mathrm{o} 3 \mathrm{~s} 33-1.40 .33 .3$

Dieser Ort weicht von Weisse IV, 383, mit welchem
Sterne Mr. Burnham A. N. 2176 den Stem identificirt, um $4^{\mathrm{s}}$ in Rectascension und 2.4 in Declination $a \mathrm{~b}$. Kur Aufklärung des Sachverhalts habe ich am 19. und 26. .Tan. d. .J. diesc Gegend am Himmel aufgesucht. Ier in der Anmerkung zu der Meridiankreisbeobachtung vom 6. Oct. 1863 erwïlnute "viel hellere Stern, der $11^{\mathrm{s}}$ rorgeht" ist Weisse IV 376. Ich fand nun die relative Lage des in Pulkowa im Meridian beobachten Sterns gegen diesen:

1878 .Jan. $19 \Delta a=+10 \operatorname{seg} \Delta d=+3^{\prime} \mathrm{l}^{\prime \prime 4}$ $" 21 \% "+10.13 \Delta+3.2 .6$

An beiden Tugen waren die Bilder schlecht und der Stern erschien bei $170 \mathrm{f}$. Vergr. des Bahusuchers einfach. Weisse IV, 376 ist im Jahre 1851 von Challis als Vergleichstern zum d'Arrest'schen Cometen benutyt und sein Ort spiiter von Villarceau an den Meridianinstrumenten der Puriser Steruwarte \%weimal bestimmt. Reducirt man diese von Oudemans in seinem "Mimoire sur l'orbite de la Comète périodique décourerte par $\mathbf{M}$. d'Arrest" mitgetheilte Position auf 1863.0, so erhält man: $"=4^{\mathrm{h}} 18^{\mathrm{m}} 43^{\mathrm{s}} 28 \quad J=-1^{\circ} 43^{\prime} 33^{\prime \prime} 9$; so dass die 1878 ermittelte Differenz der Lage beider Sterne innerhall der Beobachtungstehler mit den im Meridiane bestimmten Orten ubereinkommt.

Strassburg 1878 .Jan. 28. 